\title{
HispanismeS
}

Revue de la Société des Hispanistes Français

$18 \mid 2021$

Murs, barrières, obstacles dans les mondes hispaniques II

\section{La place de la langue française dans l'œuvre de la poétesse catalane Felícia Fuster (1921-2012)}

El papel de la lengua francesa en la obra de la poeta catalana Felícia Fuster (1921-2012)

The place of the French language in the work of the Catalan poet Felicia Fuster (1921-2012)

\section{Sandrine Frayssinhes Ribes}

\section{(2) OpenEdition Journals}

Édition électronique

URL : https://journals.openedition.org/hispanismes/13760

DOI : 10.4000/hispanismes.13760

ISSN : 2270-0765

Éditeur

Société des Hispanistes Français

\section{Référence électronique}

Sandrine Frayssinhes Ribes, «La place de la langue française dans l'œuvre de la poétesse catalane Felícia Fuster (1921-2012)», Hispanismes [En ligne], 18| 2021, mis en ligne le 31 décembre 2021, consulté le 30 janvier 2022. URL : http://journals.openedition.org/hispanismes/13760 ; DOI : https:// doi.org/10.4000/hispanismes.13760

Ce document a été généré automatiquement le 30 janvier 2022.

\section{(c) $(1)$}

Les contenus de cette revue sont mis à disposition selon les termes de la Licence Creative Commons Attribution - Pas d'Utilisation Commerciale - Pas de Modification 4.0 International. 


\section{La place de la langue française dans l'œuvre de la poétesse catalane Felícia Fuster (1921-2012)}

El papel de la lengua francesa en la obra de la poeta catalana Felícia Fuster (1921-2012)

The place of the French language in the work of the Catalan poet Felícia Fuster (1921-2012)

\section{Sandrine Frayssinhes Ribes}

Cada dia em fereixo amb la llengua materna,
Cada nit me n'he d'anar
Cap a una altra llengua materna.
ixò que em fa escriure poemes, i fa que encara
existeixi.
Extrait du poème « La llengua materna »
(traduit du japonais par Felícia Fuster)

1 Felícia Fuster (1921-2012) est une poétesse et artiste plasticienne catalane, encore méconnue en France, alors même qu'elle a passé la majeure partie de sa vie à Paris où elle s'est installée définitivement en 1951 après avoir quitté l'Espagne franquiste, poussée par son désir de liberté et sa curiosité. C'est à Paris qu'elle a créé l'essentiel de ses œuvres plastiques, et c'est là aussi qu'elle a écrit sa production poétique, loin des cercles littéraires catalans.

2 C'est une artiste à la trajectoire quelque peu singulière si l'on s'en tient aux classifications générationnelles « classiques » : bien que née dans les années 20 , elle n'a pas initié sa carrière littéraire dans les années d'après la Guerre civile, comme Joan Brossa (1919-1998) ou Vicent Andrés Estellés (1924-1993). Felícia Fuster a commencé à être connue en même temps que les jeunes poètes des années 80 ; en effet, elle ne se révèle au public lecteur en Catalogne qu'à l'âge de soixante-trois ans avec la publication de son premier recueil intitulé Una cançó per a ningú $i$ Trenta diàlegs inútils, finaliste du 
Prix Carles Riba de poésie en 1983. Cet écart générationnel s'explique par le parcours de vie de cette femme qui a dû attendre les années 80 avant de pouvoir s'adonner pleinement à l'écriture et à la création artistique. Son premier recueil est perçu comme la naissance d'une nouvelle voix poétique originale pour les Lettres catalanes, et ce à juste titre puisque les publications ultérieures viendront le confirmer.

Le propos de cet article est de présenter Felícia Fuster sous l'angle de sa relation avec la langue française, langue du pays qu'elle a délibérément choisi pour vivre son exil. L'intérêt porté à cette question est justifié par ce choix qui a eu des conséquences sur la vie littéraire de cette artiste. C'est pourquoi je reviendrai d'abord sur le choix de Paris comme ville d'adoption et ses conséquences sur la vie de cette femme catalane, tant au niveau personnel, que professionnel et artistique. J'envisagerai ensuite le rôle que la langue française a joué dans la vie littéraire de Felícia Fuster, aussi bien grâce à son expérience de la traduction que comme langue de médiation culturelle. Je terminerai enfin en présentant divers aspects de la production poétique en français de l'écrivaine.

\section{Le choix de Paris : un lieu pour vivre et pour créer}

En 1950, à l'âge de 29 ans, Felícia Fuster, diplômée des Beaux-Arts depuis 1944, profite de l'invitation de Manuel Díez, un camarade de l'École Supérieure des Beaux-Arts de Sant Jordi (Barcelone), pour faire en sa compagnie son premier voyage à la capitale française ; cet ami avait reçu une bourse de l'Institut Français de Barcelone pour se rendre à Paris et avait proposé à Felícia de l'accompagner. Ce premier voyage, qui, de fait, correspondait à ses aspirations comme artiste de découvrir d'autres tendances que celles de Barcelone, a été décisif pour la jeune catalane qui, dès son retour à Barcelone, a été convaincue qu'elle devait s'installer à Paris. Comme elle avait appris le français pendant ses années de lycée, à l'Institut Joan Maragall, la langue française ne représentait pas un obstacle à son projet. Ainsi, c'est dès 1951 qu'elle quitte l'Espagne franquiste et s'installe définitivement à Paris où en 1954, elle se marie avec un français. Ce mariage semble ne pas avoir été à la hauteur de ses espérances, comme elle a pu le déclarer elle-même dans un entretien en 1988 : « Al 54 em vaig casar i les coses no van sortir com es podia desitjar $»^{2}$. Son divorce en 1961 ouvre une nouvelle étape dans sa vie. Elle fait le choix de rester à Paris où, après avoir enseigné l'espagnol dans une école, elle travaille pour diverses agences de publicité, de 1961 à 1970. Son intégration dans le monde du travail en France ne lui a posé aucun problème. Elle a d'ailleurs suivi des études supérieures d'économie à Paris, pour obtenir en 1970 le Diplôme d'Études Supérieures Économiques du Conservatoire National des Arts et Métiers, qui lui permet de créer sa propre entreprise ${ }^{3}$. Elle arrête enfin de travailler en 1981.

5 À partir de cette date, elle consacre tout son temps à l'écriture et à l'activité artistique, comme la peinture, qu'elle peut développer dans son propre atelier. À ce moment de sa vie, elle ne se pose pas la question de savoir si elle va revenir en Catalogne, considérant qu'elle a construit toute sa vie à Paris. Son choix est surtout guidé par le fait que Paris est une ville d'art et d'artistes, une ville de tous les possibles dans le domaine artistique. Ainsi, en 1988, lors de l'entretien déjà cité, elle déclare :

[L]'ambient cultural de París és molt i molt important. Tot hi passa, a París. [...]. Hi ha un ambient de creativitat que invita a treballar. Quan tens un projecte, el pots resoldre fàcilment. Ara he tingut un problema mecànic i allà l'he pogut resoldre. Aquí no ho hauria pogut fer. I, per acabar, tinc un taller on pinto amb unes condicions de llum perfectes. Ho tinc tot organitzat a París. No puc deixar-lo4 

atelier de gravure sur verre, et elle pratique aussi la peinture sur soie et le collage ; elle faisait alors partie d'un groupe de peintres qui se sont intéressés à son travail, l'aidant à exposer aux États-Unis en 1959. Elle a participé régulièrement, dès 1958, à divers salons dédiés à l'art qui étaient organisés à Paris, et elle a réalisé plusieurs expositions dans la capitale française, notamment de son œuvre Plurivisions au début des années 90, à la Galerie Muscade et au Cloître des Billettes ${ }^{5}$.

7 Son installation à Paris a bien entendu des conséquences directes sur son éloignement des cercles littéraires catalans. Mais cela correspond de fait à un choix personnel d'évoluer en marge des mouvements artistiques et littéraires; elle revendique à la fois cette indépendance et son éclectisme, qui lui viennent, explique-t-elle, de son expérience de vie parisienne :

M'agrada continuar al marge dels moviments d'ací. És una cosa de la qual m'alegro. Jo estic a París i estic molt contenta de no formar part de cap grup. Els valors, cadascú té els seus. Així quedo al marge de tothom i va bé ser al marge. Per a mi, l'important és l'art i la llengua. Pel que fa a la resta, cadascú té els seus valors. Que sigui d'un costat o de l'altre no té importància. Sóc molt eclèctica. L'art i la persona, si val, que vingui d'allà d'on vingui, tant em fa. Això m'ha vingut de París. A París s'està per sobre de moltes coses ${ }^{6}$.

Toutefois ce profond attachement à sa ville d'adoption, Paris, ne l'a pas empêchée de garder des liens avec sa Catalogne natale, et en particulier Barcelone. Étant née dans le quartier de la Barceloneta, elle y revenait régulièrement ; en effet, elle y louait un appartement avec vue sur la mer, car c'était la seule chose qui lui manquait à Paris : «El mar és l'única cosa que em falta a París. Tota la resta és completa ${ }^{7}$. Ses séjours étaient aussi l'occasion d'entretenir ses liens d'amitié, en particulier avec des écrivains catalans, parmi lesquels la poétesse Maria-Mercè Marçal.

9 Même dans l'éloignement, Felícia Fuster a donc toujours gardé ses liens avec sa langue maternelle, qui est pour l'essentiel celle de ses poèmes; mais on peut affirmer que le français est vraiment devenu très vite pour elle sa nouvelle langue : langue de la vie quotidienne, du travail, des études et langue littéraire. Felícia Fuster, dont la curiosité intellectuelle est soulignée par ses proches et amis, a toujours manifesté un grand intérêt pour approfondir sa connaissance de la culture et de la langue françaises, au point qu'elle va se lancer dans le défi de la traduction littéraire.

\section{Felícia Fuster, traductrice du français en catalan}

Pour la poétesse catalane, l'expérience de la traduction commence dès le début des années 80. En effet, en 1984, elle publie sa version en catalan du roman historique intitulé L'œuvre au noir de Marguerite Yourcenar, roman qui avait obtenu en France en 1968 le prix Femina de littérature. La traduction est publiée en Catalogne sous le titre Obra negra ${ }^{8}$, aux éditions Proa de Barcelone. En 1982, ce texte de M. Yourcenar avait été traduit pour la seconde fois en espagnol par Emma Calatayud sous le titre Opus nigrum y Fuegos $^{9}$, la première traduction en castillan datant de $1970^{10}$. D'après l'article «La réception critique de Marguerite Yourcenar en Espagne ${ }^{11}$ de Claude Benoît, c'est au début des années 80 que des études critiques sur l'écrivaine belge ont commencé à voir le jour en Espagne, de même que les premières traductions de ses œuvres. Et L'œuvre au 
noir fut la première œuvre de M. Yourcenar à être traduite en espagnol dès 1970 , d'après l'étude de Luisa Cotoner ${ }^{12}$.

L'initiative de traduire en catalan cette auteure belge relevait, semble-t-il d'un projet personnel de Felícia Fuster, et ne répondait à aucune commande éditoriale. Elle éprouvait vraisemblablement une certaine admiration pour le profil littéraire de Marguerite Yourcenar, et elle a donc contribué à ce mouvement d'intérêt manifesté pour cette écrivaine et à « l'esforç per incorporar al cànon de la literatura catalana una autora de la talla de Marguerite Yourcenar ${ }^{13}$, selon l'étude de Luisa Cotoner citée. Felícia Fuster fait ainsi partie des onze traductrices en catalan des œuvres de Yourcenar, qui d'ailleurs ont été traduites majoritairement par des femmes selon cette même étude ${ }^{14}$. L'écrivaine belge, qui avait quelques contacts avec les milieux littéraires catalans, a été mise au courant de cette traduction catalane et a adressé, par carte

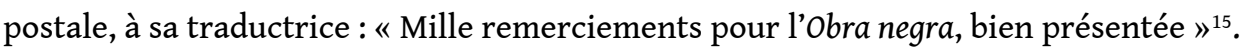

Il n'y a aucun doute sur le fait que l'intention de l'écrivaine catalane était véritablement littéraire et relevait de cette même volonté de contribuer à la construction de la généalogie féminine à laquelle aspirait la poétesse Maria-Mercè Marçal avec qui Felícia Fuster était devenue très amie depuis $1983^{16}$, comme elle l'a déclaré dans un entretien ${ }^{17}$. C'est une période où elles s'écrivaient beaucoup et où elles se sont rencontrées à diverses reprises soit à Barcelone soit à Paris, où Maria-Mercè Marçal se rendait notamment pour effectuer des recherches pour l'écriture de son roman sur l'écrivaine Renée Vivien ${ }^{18}$. Les deux poétesses catalanes ont très certainement eu de nombreux échanges au sujet de la littérature en langue française et de sa traduction en catalan. D'ailleurs, Maria-Mercè Marçal a aussi traduit Marguerite Yourcenar en publiant en 1990 la version catalane de son roman Le coup de grâce (1939) ${ }^{19}$. Cette amitié et sa bonne connaissance du français ont même conduit Felícia Fuster à relire et à amender la traduction catalane écrite par Maria-Mercè Marçal du texte de l'artiste peintre surréaliste Leonor Fini L'Oneiropompe, et publiée en $1992^{20}$.

13 Afin d'affiner le profil de traductrice de Felícia Fuster, il est intéressant d'observer quelle a été la réception de sa traduction de L'ouvre au noir. Dans son article précédemment cité, Luisa Cotoner consacre une partie à la réception des traductions catalanes dans la presse de Catalogne ${ }^{21}$; il en ressort que celle de Felícia Fuster est considérée comme excellente et fait partie de la liste des « versions excellents de gran part dels títols bàsics de l'obra d'aquesta escriptora » que Joan Josep Isern a établie ${ }^{22}$. Elle est aussi qualifiée de magnifique par Ramon Pla i Arxéz ${ }^{23}$, même s'il ne fait pas de commentaire sur le travail de traduction. Par ailleurs, l'étude de traductologie de Jesús Cabello García intitulée "Archaïsmes et formes vieillies dans L'CEuvre au Noir de Marguerite Yourcenar. Deux traductions espagnoles et une catalane ${ }^{24}$, montrent que les solutions de la version en catalan sont judicieuses, et souvent meilleures que celles des deux traductions espagnoles. Felícia Fuster a donc relevé un défi en réalisant cette traduction car elle a su résoudre les difficultés dans la transposition des archaïsmes et du lexique vieilli présents dans ce roman historique de Marguerite Yourcenar qui a choisi volontairement ce vocabulaire pour respecter les usages de la langue de la Renaissance. Et l'écrivaine catalane, tout comme la romancière, semble avoir réussi à donner au texte une sorte de " couleur " propre au XVI ${ }^{\mathrm{e}}$ siècle.

Il est évident que l'expérience de cette traduction d'une grande complexité lexicale a contribué non seulement à enrichir la connaissance de la langue française de 
l'écrivaine catalane, mais aussi à lui laisser certainement des souvenirs et des traces que l'on pourra retrouver dans sa propre poésie.

Après avoir évoqué cette première facette de Felícia Fuster comme traductrice du français, je vais aborder la question du rôle que la langue française a joué dans sa vie littéraire comme langue de médiation culturelle.

\section{Le français comme langue de médiation culturelle}

Dans les années 80, l'écrivaine catalane voue une véritable passion à la poésie japonaise. En 1985, la poétesse effectue un voyage au Japon qu'elle a préparé en amont, puisqu'elle a appris le japonais à Paris bien avant son départ, un ou deux ans avant, semble-t-il. Ce séjour au Japon, selon ses propres mots, a représenté pour elle une sorte de voyage initiatique, dans le sens où cela lui a permis de discerner de nouvelles perspectives et donc d'enrichir son écriture grâce notamment à la découverte de la poésie japonaise qu'elle commence à étudier et à traduire. En effet, en 1988, elle publie un essai sur la poésie japonaise intitulé «La poesia japonesa moderna»dans la revue Revista de Catalunya ${ }^{25}$. La même année, elle publie en catalan une anthologie de poèmes japonais $\mathrm{du} \mathrm{XX} \mathrm{X}^{\mathrm{e}}$ siècle ${ }^{26}$, éditée par la maison d'édition Proa, contribuant ainsi à la connaissance de cette poésie en Catalogne grâce à la traduction de poèmes de quarante-neuf auteurs différents encore inédits en catalan, selon Mercè Altimir ${ }^{27}$; il s'agit d'écrivains nés entre la fin du XIX siècle et les années 50 . Cette traduction est le fruit d'une collaboration avec un étudiant japonais, Naoyuki Sawada, qui étudiait alors la philosophie à la Sorbonne, et est aujourd'hui professeur à l'université de Rikkyo au Japon; il a récemment expliqué lors d'une communication qu'il s'agissait d'une double traduction car lui-même traduisait les textes japonais en français et Felícia Fuster les réécrivait ensuite en catalan ${ }^{28}$.

Il est intéressant d'observer que Felícia Fuster publie son essai et ses traductions dans le contexte particulier de la fin des années 80, époque où se manifeste en France un intérêt marqué pour la poésie japonaise. Cette période est, en effet, riche en publications de traductions de haikus notamment. Et c'est par le biais des nombreuses traductions françaises que Felícia Fuster découvre dans un premier temps la poésie japonaise et qu'elle a accès aux formes poétiques orientales que sont le tanka et le haiku. Signalons ici l'anthologie publiée en 1978 par Maurice Coyaud ${ }^{29}$ qui a non seulement fait connaître des haikus japonais, mais a aussi explicité l'esprit du haiku pour le public français ; à partir de cette date, l'intérêt des poètes et des traducteurs français pour le genre est allé croissant. Pour la poétesse catalane, le français se pose alors en langue de médiation pour la connaissance de cette littérature orientale. Lorsque l'on connaît sa grande curiosité intellectuelle, on imagine aisément le besoin qu'elle a pu éprouver d'apprendre le japonais afin d'appréhender directement la signification des poèmes.

Parmi les traducteurs du japonais, Felícia Fuster a connu le Professeur René Sieffert ${ }^{30}$ (1923-2004), enseignant de japonais à Paris, président de l'Institut National des Langues et Civilisations Orientales (INALCO), spécialiste des formes poétiques japonaises en France, et traducteur de l'essentiel des publications de Bashô dont Le haikai selon Bashô (1983). René Sieffert a contribué à rapprocher la littérature japonaise des lecteurs francophones. À la fin de son prologue pour l'ouvrage Poesia japonesa contemporània, Felícia Fuster remercie en particulier ce professeur de l'aide qu'il lui a apportée pour 
finaliser ses traductions: "vull mencionar especialment el professor René Sieffert, director del Departament de Llengües Orientals de París, que amb tanta senzillesa m'ha acollit. Els seus valuosos consells i observacions m'han permès d'establir la versió final de molts poemes $»^{31}$. Ce spécialiste a reçu à diverses reprises l'écrivaine catalane pour lui donner des conseils pour la traduction des poèmes japonais. Leur langue de communication était le français, et c'est donc par la médiation de la langue française que Felícia Fuster a pu affiner ses traductions en catalan. Elle a tissé des liens professionnels et amicaux avec ce professeur français avec qui elle a pu partager sa passion pour la culture japonaise; elle a même eu l'occasion de l'inviter à l'inauguration de l'une de ses expositions de ses œuvres plastiques.

Même s'il est certain que la langue française a joué un rôle dans son approche des poèmes japonais, il n'en est pas moins sûr que la traduction des poèmes japonais contemporains en catalan dans son anthologie de 1988 est de première main, comme elle l'affirme elle-même dans son prologue où elle précise bien qu'il s'agit d'une traduction directe des textes japonais en catalan : « aquesta traducció catalana directa dels textos japonesos $»^{32}$. En effet, la plupart des traductions publiées en France à cette date et auxquelles Felícia Fuster a eu accès - ou aurait pu avoir accès - sont des poèmes d'auteurs japonais anciens, et non de poètes contemporains, lesquels ont été traduits en français et publiés beaucoup plus tard, dans les années 2000 seulement. Selon Jean Antonini, spécialiste et traducteur en français de la poésie japonaise, «les premiers poèmes japonais contemporains en français apparaissent dans la seconde anthologie de Corine Atlan et Zéno Bianu, et dans les anthologies présentées par Dominique Chipot et Makoto Kemmoku »33. C'est donc dans l'anthologie Haiku du XXe siècle, le poème court japonais d'aujourd'hui ${ }^{34}$, publiée en 2007, que l'on peut lire les premiers haïkus japonais écrits au XXe siècle traduits en français. Et en 2008, l'anthologie bilingue intitulée $D u$ rouge aux lèvres ${ }^{35}$ permet de découvrir aussi d'autres auteurs japonais et japonaises du $\mathrm{XX}^{\mathrm{e}}$ siècle, aux côtés des classiques. Ensuite, « les deux premières publications dédiées complètement à des poètes japonais contemporains ont lieu en 2013 et $2014 »^{36}$ seulement, avec la traduction de Corine Atlan intitulée Haikus du temps présent ${ }^{37}$, et celle de J. Antonini et K.Tajima sous le titre Cascade du futur ${ }^{38}$.

Ces données chronologiques sont importantes car Felícia Fuster publie en 1990 en France une anthologie de quatre-vingt-un poèmes japonais traduits en français dans la revue de poésie Vagabondages ${ }^{39}$. Il s'agit d'une sélection de textes appartenant à la poésie japonaise dont la plupart avaient déjà été traduits en français dans diverses publications ; Felícia Fuster y ajoute quatre poèmes du XX $\mathrm{XX}^{\mathrm{e}}$ siècle ${ }^{40}$ qu'elle cotraduit en français avec Naoyuki Sawada, l'étudiant japonais qui l'avait déjà aidée pour les traductions en catalan ${ }^{41}$. Ainsi, grâce à l'anthologie en français, l'écrivaine catalane a contribué au rapprochement entre la poésie japonaise et les lecteurs français.

21 Son expérience de la traduction de la poésie japonaise montre que la langue française a joué un rôle primordial dans son travail de traductrice car elle lui a permis non seulement de découvrir la poésie japonaise classique, grâce aux traductions publiées dans les années 70-80 en France, de partager sa passion pour cette littérature orientale avec des spécialistes, mais aussi de contribuer à la réception et à la diffusion de la poésie japonaise, aussi bien en Catalogne qu'en France.

22 Cet attachement littéraire de Felícia Fuster à la langue française qu'elle s'est appropriée comme traductrice pendant les années 80-90, l'a même conduite à faire le choix d'écrire des poèmes en langue française. 


\section{Le français comme langue de création poétique} artistiques ou littéraires ; d'ailleurs, dans un entretien radiophonique ${ }^{42}$ de 1984, elle affirme qu'elle n'a pas de contact à Paris en lien avec la poésie et qu'elle vit loin de tout, sans contact non plus avec d'autres écrivains exilés. Cependant, même si elle préférait rester en marge, il est évident que sa poésie relève de l'expérimentation formelle et qu'elle explore les possibilités créatrices du langage comme l'ont fait les poètes français surréalistes de l'avant-garde, afin d'exprimer l'essence poétique de l'expérience intériorisée, comme le souligne Francesc Parcerisas lorsqu'il définit la poésie de Felícia Fuster dans le prologue du recueil Aquelles cordes del vent ${ }^{43}$ (1987) : « Ens trobem davant una poesia despullada perquè tota ella és destinada a la recerca de l'essència interioritzada, d'allò que hi ha darrere l'experiència no com a tal experiencia, sinó com a vivència susceptible d'ésser traslladada, mitjançant la poesia, a una forma del llenguatge $»^{44}$. Certains critiques catalans, dont Parcerisas dans ce même prologue, ont établi un lien entre les poèmes de la poétesse et la poésie française contemporaine, notamment Paul Éluard et René Char. Ce rapprochement se justifie en particulier par la recherche sur le vers libre. Il ne faut pas oublier que cette femme est aussi une artiste plasticienne qui expérimente des techniques et qu'elle a donc une sensibilité particulière pour la recherche et l'expérimentation formelle qui correspond, de fait, à cette littérature innovante, libre, radicale qui s'est constituée avec les avant-gardes. Le concept de liberté est au cœur de ce mouvement de la poésie du début du XX $\mathrm{XX}^{\mathrm{e}}$ siècle. D'ailleurs, c'est le même message que l'artiste catalane propose dans ses œuvres plastiques et ses poèmes: un "message de liberté " selon ses propres mots: «M'agradaria que es recordés la meva obra pel missatge de llibertat que dóna i de superació de la solitud $»^{45}$.

24 Ainsi Felícia Fuster manifeste un intérêt particulier pour les formes proposées par les avant-gardes qu'elle explore dans un premier temps, pour peu à peu s'en libérer et créer ses propres formes issues de sa recherche sur le langage. Elle emprunte notamment à diverses reprises le calligramme, forme poétique qu'elle se réapproprie, car, pour elle, il est l'association idéale des mots écrits et de l'image. Dans l'histoire de la poésie, le calligramme est une forme d'écriture identifiée comme française et il est associé à un nom, celui de Guillaume Apollinaire (1880-1918), considéré comme précurseur du surréalisme.

C'est dans la seconde moitié des années 80 que Felícia Fuster revisite la forme du calligramme. Elle réalise deux livres d'artiste qu'elle édite sous le nom éditorial de "Livre libre", selon la technique de la sérigraphie, et en édition limitée de 30 exemplaires chacun. Ils s'intitulent en français : Écume fêlée (1986) et Au bout des os au bout des mots (1989). Ce sont quelques textes calligraphiés rédigés en français et illustrés. 
Figure 1 : extrait du livre d'artiste Écume fêlée

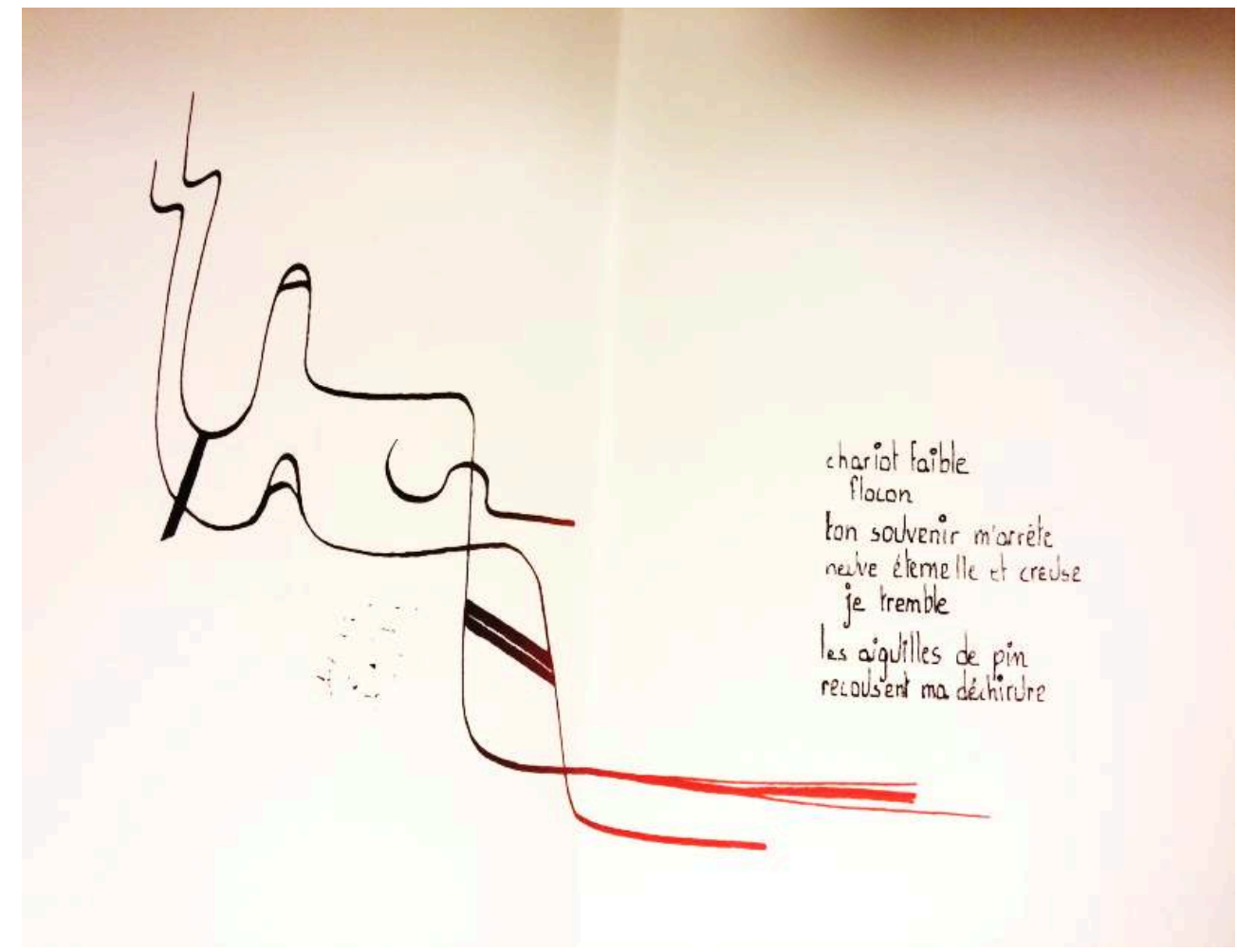

Source : cliché personnel

Dans un entretien de 1988, l'écrivaine parle du projet de livre d'artiste de 1989 en soulignant le fait que c'est elle qui a écrit les poèmes en français qui accompagneront les dessins : "Serà un llibre amb dibuixos i poemes en francès, poemes escrits per mi directament en francés ${ }^{46}$. Dans Écume fêlée (1986) (figure 1), la partie de texte adopte la forme d'un calligramme, puisque les mots dessinent un tracé pouvant évoquer une image, mais le texte est accompagné d'un dessin. Dans Au bout des os au bout des mots (1989) (figure 2), les textes sont vraisemblablement écrits selon la méthode de l'écriture automatique - qui associe des mots émergeant de l'inconscient - et la poétesse joue sur les sonorités. À l'image de la poésie surréaliste, Felícia Fuster adopte dans ces réalisations la ligne expérimentale de destruction de la conception classique de l'écriture, en omettant la ponctuation, en privilégiant les allitérations et le rythme. 
Figure 2 : extrait du livre d'artiste Au bout des os au bout des mots

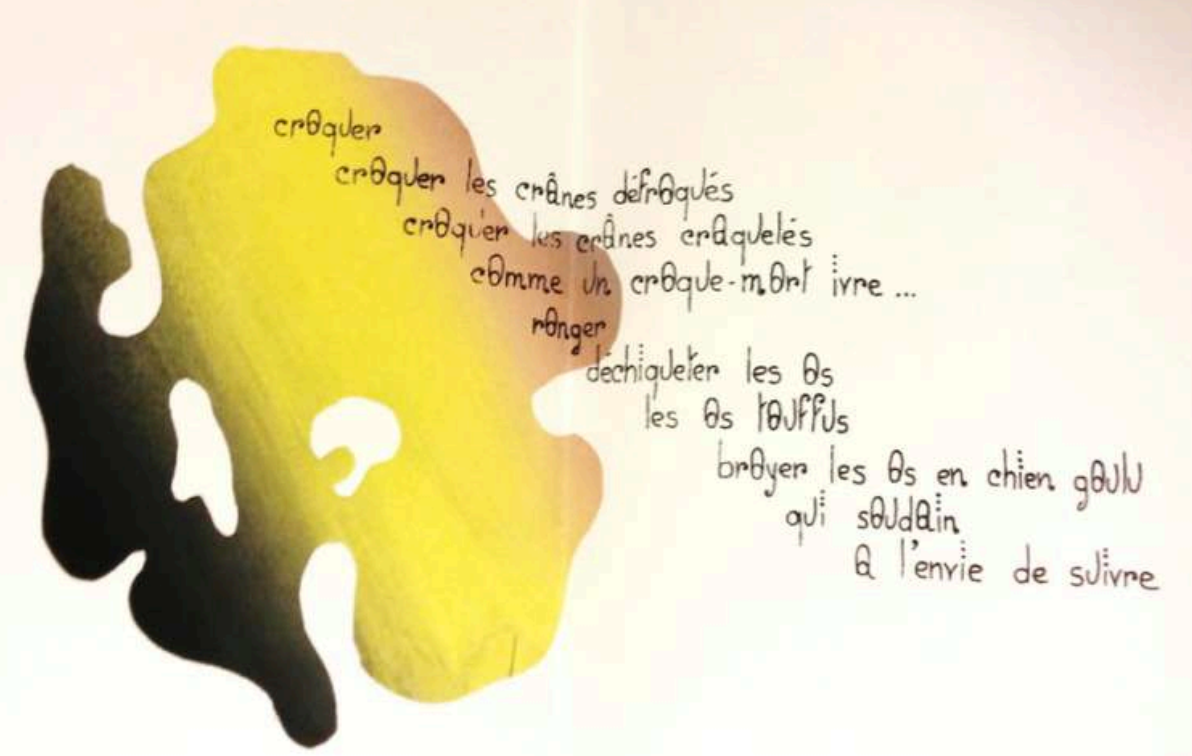

Source : cliché personnel

En 1992, Felícia Fuster édite à Barcelone une plaquette intitulée Passarel-les / Mosaïques ${ }^{47}$ écrite en catalan et en français. Les textes présentés comme les Mosaïques sont trois calligrammes écrits en français. L'auteure a choisi le mot français Mosaïques pour désigner ces trois poèmes; c'est un terme qui renvoie à une autre pratique artistique que celle de la poésie, ce qui n'est pas étonnant chez cette artiste sensible aux liens qui s'établissent entre les mots et la perception visuelle. Mosaïques est le seul recueil de Fuster publié en français. Cette fois, les calligrammes édités sont des textes non pas calligraphiés, mais écrits à la machine. Chacun représente par sa forme un objet en lien avec la signification du texte. Voici le premier des trois : 


\title{
Jarres douces de vin nous étions sur la rive ruban d'argile vive l'eau passait sous nos pieds le temps semblait solide et le vide effacé nos mains tenaient le large
}

\author{
Rien n'est jamais gagné Jarres pleines \\ de fiel nous étions sur la rive
}

\section{ruban d'argile argile argile froide l'eau passait}

Extrait de Felícia Fuster, Obra poètica, p. 217.

La poétesse catalane s'affranchira du lien du calligramme avec la langue française pour créer de nouveaux calligrammes en catalan dans son recueil sorra de temps absent (1998) où elle développera cette forme, jouant avec la typographie, l'usage des majuscules, la mise en page, les espaces en blanc, l'absence de ponctuation, le vers libre. Chaque texte est, comme l'écrit Lluïsa Julià, un " poema dibuixat en el full de paper ${ }^{48}$. La poétesse elle-même s'exprime ainsi à ce propos : « la meva poesia busca formes visuals, com és el cas del recull Sorra de temps absent. El poema "De massa", per exemple, representa un gra de sorra, el gra de sorra que som, de massa $»^{49}$. Ce volume Sorra de temps absent, que d'aucuns considèrent comme l'œuvre la plus ambitieuse de Felícia Fuster, lui a valu d'être finaliste du Prix Màrius Torres en 1997.

L'abandon du calligramme en français ne signifie pas pour autant que la poétesse n'écrira plus de poésie dans sa langue d'adoption. En effet, le 8 février 1993, Felícia Fuster est l'invitée de Núria Anglada lors de son programme radiophonique ${ }^{50}$ culturel diffusé sur Radio Pays dans le Roussillon. Cet entretien se déroule essentiellement en français, mais aussi pour une partie en catalan, puisque aussi bien la présentatrice que l'écrivaine passent d'une langue à l'autre, avec des lectures de poèmes de l'invitée publiés en catalan, qui sont traduits et commentés en français en direct pour faciliter la compréhension du public non catalanophone. Cette émission radiophonique est aussi l'occasion pour l'artiste de lire deux poèmes inédits qu'elle a écrits en français en lien avec le thème de la mer. N'ayant pas eu accès à ces textes originaux, je propose ici la transcription de ces deux poèmes, que j'ai réalisée selon le rythme de la lecture suivi par Felícia Fuster à l'antenne.

Premier poème :

Paradis faible de la chair

où se brise

l'ogive de la voile

Le portulan des songes

Second poème : 
Perdu dans le ressac

du propre labyrinthe, que faire

pour déjouer les cordes

et lire

les tournants de la houle

vers 1 et 4 , mètre qui fut utilisé par Verlaine, poète symboliste, et dans le second texte, l'usage d'hexasyllabes aux vers 1, 2, 4 et 6. L'hexamètre fut aussi choisi par Verlaine et Robert Desnos, surréaliste. Le choix particulier de ces mètres avec leur relative régularité ainsi que le jeu sur les sonorités témoignent d'une part de la bonne connaissance de la poésie française, en particulier de la pratique poétique de l'avantgarde et du surréalisme, et d'autre part de la sensibilité de Felícia Fuster aux sonorités de la langue française. Cette connaissance passe aussi par celle du lexique, notamment celui en lien avec le monde maritime dans ces deux poèmes. Dans l'entretien, l'écrivaine explique en français ce que signifie le mot « portulan » qu'elle a emprunté à Marguerite Yourcenar, laquelle l'utilise dans son roman L'œuvre au noir traduit en catalan par Felícia Fuster. Le fait que ce terme, d'un emploi très rare, n'apparaisse qu'une seule fois dans ce roman souligne l'extrême sensibilité aux mots de la poétesse qui explique en français dans l'entretien radiophonique que ce second poème consiste à «trouver le port où il $\mathrm{y}$ a le plus beau rêve ». En commentant ses poèmes, elle affirme que ce n'est pas une "poésie linéaire " mais " une poésie à plans divers comme un paysage » et elle explique que les poèmes se construisent de la même façon que la peinture. Cette notion d'une poésie par plans correspond à l'évolution de sa conception de l'écriture poétique qu'elle tient essentiellement de son expérience de lecture et de traduction de la poésie japonaise ; en effet, elle relève déjà en 1988 dans le prologue de son anthologie Poesia japonesa contemporània cette caractéristique nouvelle de la poésie japonaise dite moderne ${ }^{51}$ qui est liée à l'idée d'image filmique utilisant des plans différents :

Una característica nova és potser el que podríem anomenar carácter visual, cinematogràfic, del poema, cosa que ja existia en potencia en el haiku, però només com a evocació. Les poesies no són narratives, no descriuen, ens fan veure amb els nostres ulls, mirar unes escenes precises, i focalitzen amb els versos detalls, com en un primer pla, o ens porten de nou a un pla general ${ }^{52}$.

33

L'analyse de la production poétique en langue française de Felícia Fuster que nous avons évoquée montre que pour elle, ce qui compte en poésie c'est d'abord l'effet produit à la fois sur le plan rythmique et au niveau visuel. Peu importe la langue d'expression choisie ; en effet, elle met en pratique cette même conception de l'écriture que ce soit dans sa langue maternelle ou en français.

Il est indéniable que des liens forts se sont tissés entre la poétesse catalane et la langue française au fil des années qu'elle a passées à Paris, loin de sa Catalogne natale. Langue d'adoption, le français est vite devenu pour Felícia Fuster une langue de médiation littéraire entre les trois cultures qui l'ont forgée comme écrivaine et poétesse. Et même si à ce jour, aucun autre texte écrit en français que ceux que j'ai présentés ici n'a fait l'objet d'une étude, ou d'une publication ou de toute autre forme de diffusion, il apparait que pour cette femme catalane le français pouvait aussi être une langue d'écriture et de création. Son excellente connaissance de la langue et de la poésie françaises lui a ouvert des voies nouvelles dans l'exploration de sa propre langue et du langage poétique, constituant un apport décisif dans son œuvre. Sa recherche sur le 
langage rejoint ses préoccupations artistiques et fait de cette poétesse une voix originale en Catalogne.

Pour finir, je vais aborder un point qui me semble central pour cette année où est célébré le centenaire de sa naissance : celui de la réception en France de son œuvre littéraire. Jusqu'en 2018, aucun de ses recueils de poèmes n'avait été traduit en français. L'anthologie bilingue Cordages $d u$ vent ${ }^{53}$ éditée par les Editions Trabucaire est la première publication de traductions en français de poèmes de cette artiste aux multiples facettes. Ce projet de traduction en France est une sorte de retour symbolique de Felícia Fuster vers sa terre d'accueil. Il est né de la volonté de Lluïsa Julià, critique littéraire barcelonaise et spécialiste de la littérature écrite en catalan par des femmes, de faire connaître Felícia Fuster en France. C'est au professeur Marc Audí, spécialiste de poésie catalane, que l'on doit cette première traduction en français qui relève le défi d'être en concordance avec la conception de la traduction de la poétesse catalane pour qui il est important de « conserver un rythme » pour être le plus fidèle à «l'essence des poèmes $\|^{54}$. Alors même que le centenaire de la naissance de Felícia Fuster est l'occasion d'organiser en France des événements comme la lecture bilingue ${ }^{55}$ de ses poèmes, qui a eu lieu le 6 mai 2021, et qui était précédée de communications sur l'artiste ${ }^{56}$, et également un séminaire interuniversitaire ${ }^{57}$ ayant pour objectif de visibiliser son œuvre, nous ne pouvons désormais qu'espérer qu'un projet de traduction de l'ensemble de l'œuvre poétique de Felícia Fuster puisse voir le jour en France pour contribuer à ce mouvement de circulation de la culture de l'Autre auquel cette écrivaine a elle-même concouru, alimentant la dimension créative de la traduction.

\section{BIBLIOGRAPHIE}

Nora ANCAROLA, Lluïsa JULIÀ, «Felícia Fuster, poeta i pintora», Avui, nº du 15/03/2001, (2001), p. XXI.

Nora ANCAROLA, Lola DONAIRE (coords.), Felícia Fuster. Obra plàstica, Barcelona, Fundació Felícia Fuster, 2008.

Mercè ALTIMIR, «Felícia Fuster», Visat [on-line], n¹8 (2014) [consulté le 25/05/2021] <URL: http:// www.visat.cat/espai-traductors/esp/traductor/351/felicia-fuster.html>.

Corine ATLAN, Zéno BIANU, Haiku du XXe siècle, le poème court japonais d'aujourd'hui, Paris, Gallimard, 2007.

Claude BENOIT, «La réception critique de Marguerite Yourcenar en Espagne », Réception critique de l'œuvre de Marguerite Yourcenar, Actes du colloque international de Clermont-Ferrand (22-24 novembre 2007), 2007 p. 111-121.

Antoni BONADA, «El sol ix per a Felícia Fuster», El Temps, n¹88 (1988), p. 64-66.

Jesús CABELLO GARCíA, « Archaïsmes et formes vieillies dans L'CEuvre au Noir de Marguerite Yourcenar. Deux traductions espagnoles et une catalane », Dialogues interlinguistiques, CELTA/ CoVariUs, Université Paris Sorbonne, 2008, p.1.1. 
Dominique СНIPOT, Makoto KЕмMокU, Du rouge aux lèvres, Paris, La Table Ronde, 2008.

Dominique CHIPOT, Jean ANTONINI, Histoire du haiku en France [on-line], 2014, p. 15, [consulté le 25/05/2021] < URL: https://www.thehaikufoundation.org>.

Luisa COTONER, «Les traduccions al català de Marguerite Yourcenar: edicions i recepció crítica», Revista de Filología Románica, vol. 29, n² (2012), p. 275-289.

Maurice COYAUD, Fourmis sans ombre, le livre du haiku, Paris, Phébus Libretto, 1978.

Leonor FINI, L'Oneiropompe (Traducció de Maria-Mercè Marçal, revisió de Felícia Fuster),

Barcelona, Edicions de l'Eixample, 1992.

Felícia FUSTER, «Entrevista a Felícia Fuster», Veus i poemes (présenté par Alexandra), Ràdio Molins de Rei, 07/03/1984. Durée 60 minutes (consulté aux archives de la Fundació Felícia Fuster, Barcelona).

Felícia FUSTER, Aquelles cordes del vent, Barcelona, Proa, 1987.

Felícia FUSTER, «La poesia japonesa moderna», Revista de Catalunya, n¹9 (mai 1988), p. 131-150.

Felícia FUSTER (ed.), Poesia japonesa contemporània (Traducció de Felícia Fuster i Naoyuki Sawada), Barcelona, Proa, 1988.

Felícia FUSTER, « Poésie japonaise », Vagabondages, revue de poésie, n77 (1990).

Felícia FUSTER, Passarel·les/Mosaïques (plaquette o plec de poesia en català i francès) Barcelona, Cafè Central, 1992.

Felícia FUSTER, « Entretien de Núria Anglada avec Felícia Fuster », Emissions catalanes, Radio Pays, 18/02/1993. Durée 1h33 (consulté aux archives de la Fundació Felícia Fuster, Barcelona).

Felícia FUSTER, Obra poètica, Barcelona, Proa, 2010.

Felícia FUSTER, Cordages du vent. Anthologie bilingue de poèmes (Traduction de Marc Audí ; Édition de Lluïsa Julià et Sandrine Frayssinhes Ribes), Canet de Rosselló, Perpignan, Trabucaire, 2018.

Joan Josep ISERN, «Yourcenar: la grandesa de la miniatura. 'Contes orientals', un llibre de lenta elaboració», Avui. Cultura, nº du 20/01 (1990).

Lluïsa JULIÀ, «Pròleg», Felícia FUSTER, Obra poètica, Barcelona, Proa, 2010, p. 9-18.

Lluïsa, JULIÀ, «Dues veus singulars: la relació entre Maria-Mercè Marçal i Felícia Fuster», Heura Marçal (ed.), V Jornades marçalianes, Sabadell, Fundació Maria-Mercè Marçal, 2014, p. 135-151.

Mayuzumi МАDоКА, Haikus du temps présent (sélection et présentation de la traductrice Corine Atlan), Arles, Picquier, 2012.

Maria-Mercè MARÇAL, La passió segons Renée Vivien, Barcelona, Columna, 1994 (1ère éd.).

Ban'ya NATSUISHI, Cascade du futur (traduction du japonais par Jean Antonini et Keiko Tajima), Paris, L'Harmattan, 2014.

Ramon PLA I ARXÉ, «Passió freda», Avui, nº du 12/09 (1996).

Marguerite YOURCENAR, El Alquimista (Traducción de Vicente Villacampa), Barcelona, Plaza \& Janés, 1970.

Marguerite YOURCENAR, Opus nigrum (Traducción de Emma Calatayud), Madrid, Alfaguara, 1982. Marguerite YOURCENAR, Obra negra (Traducció de Felícia Fuster), Barcelona, Proa, 1984. 
Marguerite YouRCENAR, Carte postale adressée à Felícia Fuster, datée de décembre 1985 (consultée aux archives de la Fundació Felícia Fuster, Barcelona).

Marguerite YOURCENAR, El tret de gràcia (Traducció de Maria-Mercè Marçal), Barcelona, Edicions del Mall, 1990.

\section{NOTES}

1. Felícia FUSTER, Obra poètica, Barcelona, Proa, 2010, p. 442.

2. Antoni BONADA, «El sol ix per a Felícia Fuster», El Temps, n¹88 (1988), p. 64-66.

3. Cf. Lluïsa, JULiÀ, «Dues veus singulars: la relació entre Maria-Mercè Marçal i Felícia Fuster», Heura Marçal (ed.), V Jornades marçalianes, Sabadell, Fundació Maria-Mercè Marçal, 2014, p. 135-151.

4. Ibid.

5. Cf. Nora ANCAROLA, Lola DONAIRE (coord.), Felícia Fuster. Obra plàstica, Barcelona, Fundació Felícia Fuster, 2008.

6. Antoni BONADA, «El sol ix per a Felícia Fuster», op. cit.

7. Ibid.

8. Marguerite YouRCENAR, Obra negra (Traducción de Felícia Fuster), Barcelona, Proa, 1984.

9. Marguerite YOURCENAR, Opus nigrum (Traducción de Emma Calatayud), Madrid, Alfaguara, 1982.

10. Marguerite yourcenAR, El Alquimista (Traducción de Vicente Villacampa), Barcelona, Plaza \& Janés, 1970.

11. Claude BENOIT, "La réception critique de Marguerite Yourcenar en Espagne ", Réception critique de l'œuvre de Marguerite Yourcenar, Actes du colloque international de Clermont-Ferrand (22-24 novembre 2007), 2007 p. 111-121.

12. Luisa COTONER, «Les traduccions al català de Marguerite Yourcenar: edicions i recepció crítica», Revista de Filología Románica, vol. 29, n² (2012), p. 275-289.

13. Ibid., p. 288.

14. Ibid., p. 284.

15. Marguerite yOURCENAR, Carte postale adressée à Felícia Fuster, datée de décembre 1985 (consulté aux archives de la Fundació Felícia Fuster, Barcelona).

16. Cf. Lluïsa, JULIÀ, «Dues veus singulars: la relació entre Maria-Mercè Marçal i Felícia Fuster», op. cit.

17. Nora ANCAROLA, Lluïsa JULIÀ, «Felícia Fuster, poeta i pintora», Avui, nº du 15/03/2001, (2001), p. X-XI.

18. Maria-Mercè MARÇAL, La passió segons Renée Vivien, Barcelona, Columna, 1994 (1ère éd.).

19. Marguerite YOURCENAR, El tret de gràcia (Traducció de Maria-Mercè Marçal), Barcelona, Edicions del Mall, 1990.

20. Leonor FINI, L'Oneiropompe (Traducció de Maria-Mercè Marçal, revisió de Felícia Fuster), Barcelona, Edicions de l'Eixample, 1992.

21. Luisa COTONER, «Les traduccions al català de Marguerite Yourcenar: edicions i recepció crítica». Op. Cit., p. 286.

22. Joan Josep ISERN, «Yourcenar: la grandesa de la miniatura. 'Contes orientals', un llibre de lenta elaboració», Avui. Cultura, nº du 20/01 (1990).

23. Ramon PLA I ARXÉ, «Passió freda», Avui, nº du 12/09 (1996).

24. Jesús CABELLO GARCíA, «Archaïsmes et formes vieillies dans L'Éuvre au Noir de Marguerite Yourcenar. Deux traductions espagnoles et une catalane ", Dialogues interlinguistiques, CELTA/ CoVariUs, Université Paris Sorbonne, 2008, p.1.1. 
25. Felícia FUSTER, «La poesia japonesa moderna», Revista de Catalunya, n¹9 (mai 1988), p. 131-150. 26. Felícia FUSTER (ed.), Poesia japonesa contemporània (Traducció de Felícia Fuster i Naoyuki Sawada), Barcelona, Proa, 1988.

27. Mercè ALTimiR, «Felícia Fuster», Visat [on-line], n¹8 (2014) [consulté le 25/05/2021] <URL:http://www.visat.cat/espai-traductors/esp/traductor/351/felicia-fuster.html>.

28. Naoyuki SAWADA, «La traduction avec Felícia Fuster : poésie japonaise et catalane ", Jornades Felícia Fuster "La pàgina és la pell», colloque organisé par l'ADHUC-Centre de Recerca Teoria, Gènere, Sexualitat, Universitat de Barcelona, 5-6 octobre 2021.

29. Maurice CoYAUD, Fourmis sans ombre, le livre du haiku, Paris, Phébus Libretto, 1978.

30. René Sieffert a créé en 1971 la maison d'édition universitaire Publications Orientalistes de France (POF).

31. Felícia FUSTER (ed.), Poesia japonesa contemporània, Op. cit., p.12.

32. Ibid., p. 11.

33. Dominique CHIPOT, Jean ANTONINI, Histoire du haiku en France [on-line], 2014, p. 15, [consulté le 25/05/2021] < URL: https://www.thehaikufoundation.org>.

34. Corine ATLAN, Zéno BIANU, Haiku du XXe siècle, le poème court japonais d'aujourd'hui, Paris, Gallimard, 2007.

35. Dominique СНIPOT, Makoto KЕмМокU, Du rouge aux lèvres, Paris, La Table Ronde, 2008.

36. Dominique CHIPOT, Jean ANTONINI, Histoire du haiku en France, Op. cit., p.15.

37. Mayuzumi МАDОКА, Haikus du temps présent (sélection et présentation de la traductrice Corine Atlan), Arles, Picquier, 2012.

38. Ban'ya NATSUISHI, Cascade du futur (traduction du japonais par Jean Antonini et Keiko Tajima), Paris, L'Harmattan, 2014.

39. Felícia FUSTER, « Poésie japonaise », Vagabondages, revue de poésie, $\mathrm{n}^{\circ} 77$ (1990).

40. Il s'agit des poèmes suivants : « Aiguilles de pin » (1920) et "Le matin de la séparation » (1924) de Miyazawa Kenji, "Le prisonnier » (1949) de Miyoshi Toyoichirô, et "Pua-Pua, la prostituée vierge fait l'AEIOU ménager » (1967) de Suzuki Shiroyasu.

41. Felícia FUSTER (ed.), Poesia japonesa contemporània, op. cit.

42. Felícia FUSTER, «Entrevista a Felícia Fuster», Veus i poemes (présenté par Alexandra), Ràdio Molins de Rei, 07/03/1984. Durée 60 minutes (consulté aux archives de la Fundació Felícia Fuster, Barcelona).

43. Felícia FUSTER, Aquelles cordes del vent, Barcelona, Proa, 1987.

44. Francesc PARCERISAS, «Pròleg», Felícia Fuster, Obra poètica, Op. cit., p. 499-502.

45. Nora ANCAROLA, Lluïsa JULIÀ, «Felícia Fuster, poeta i pintora», Op. cit., p.XI.

46. Antoni BONADA, «El sol ix per a Felícia Fuster», op. cit.

47. Felícia FUSTER, Passarel-les/Mosaïques (plaquette o plec de poesia en català i francès) Barcelona, Cafè Central, 1992.

48. Lluïsa JULIÀ, «Pròleg», Felícia FUSTER, Obra poètica, op. cit., p. 17.

49. Nora ANCAROLA, Lluïsa JULIÀ, «Felícia Fuster, poeta i pintora», op. cit.

50. Felícia FUSTER, « Entretien de Núria Anglada avec Felícia Fuster », Emissions catalanes, Radio Pays, 18/02/1993, durée 1h33 (consulté aux archives de la Fundació Felícia Fuster, Barcelona).

51. Dans le prologue de son anthologie Poesia japonesa contemporània, Fuster explique que pour les japonais, la poésie appelée «moderne " correspond à celle qui apparaît au Japon vers la fin du $\mathrm{XIX}^{\mathrm{e}}$ siècle et qui se développe surtout à partir des années 20. Cf. Felícia FUSTER, «Pròleg», Poesia japonesa contemporània, op. cit., p. 7.

52. Ibid., p. 9.

53. Felícia FUSTER, Cordages du vent. Anthologie bilingue de poèmes (Traduction de Marc Audí ; Édition de Lluïsa Julià et Sandrine Frayssinhes Ribes), Canet de Rosselló, Trabucaire, 2018. 
54. Felícia FUSTER, «Entretien de Núria Anglada avec Felícia Fuster », Emissions catalanes, Radio Pays, 18/02/1993, durée 1h33 (consulté aux archives de la Fundació Felícia Fuster, Barcelona).

55. Conférence Sobre l'obra de Felícia Fuster. Lectura bilingüe / Autour de l'oeuvre de Felícia Fuster. Lecture bilingue (06/05/2021). La lecture des poèmes a été réalisée par des étudiants des universités françaises de Perpignan Via Domitia et de Paul-Valéry Montpellier III.

56. Les communications ont été présentées par : Lluïsa Julià (Universitat Oberta de Catalunya), Caterina Riba (Universitat de Vic-Universitat Central de Catalunya), Sandrine Frayssinhes Ribes (Université Paul-Valéry Montpellier III), Mireia Vidal-Conte (poétesse).

57. Il s'agit du séminaire interuniversitaire de littérature catalane intitulé De la cryptogynie à la médiatisation des écrivaines Felícia Fuster et Carmelina Sánchez Cutillas et consacré à l'étude des œuvres de ces écrivaines nées toutes deux en 1921 (novembre 2021 - avril 2022). Séminaire organisé par les laboratoires de recherche d'universités françaises CRESEM-UPVD, LER-UP8, LLACREATIS-UT2J et ReSO-UPVM, et le groupe de recherche GETLIHC de l'Universitat de VicUniversitat Central de Catalunya.

\section{RÉSUMÉS}

C'est dans le cadre de la célébration du centenaire en 2021 de la naissance de Felícia Fuster que je me propose de présenter cette poétesse née à Barcelone en 1921, sous l'angle de sa relation avec la langue française qui a joué un rôle clé dans sa vie littéraire. Elle a fait le choix de vivre en France, à Paris dès 1951. Le français est devenu pour cette artiste catalane d'abord une langue de traduction, mais aussi de médiation culturelle et enfin de création poétique.

As part of the celebration of the centenary of Felícia Fuster's birth in 2021, I propose to present this poetess, born in Barcelona in 1921, from the point of view of her relationship with the French language, which played a key role in her literary life. She chose to live in France, in Paris, from 1951. For this Catalan artist, French became first and foremost a language of translation, but also of cultural mediation and finally of poetic creation.

\section{INDEX}

Keywords : Felícia Fuster, poetry, Catalan, French, Marguerite Yourcenar, translation

Mots-clés : Felícia Fuster, poésie, catalan, français, Marguerite Yourcenar, traduction

\section{AUTEUR}

\section{SANDRINE FRAYSSINHES RIBES}

Université Paul-Valéry Montpellier 3 